\title{
Estimation of Mediterranean forest attributes by the application of k-NN procedures to multitemporal Landsat ETM+ images
}

\author{
F. MASELLI†, G. CHIRICI†, L. BOTTAI§, P. CORONA $\uparrow$ and \\ M. MARCHETTI** \\ $\dagger$ IBIMET, Consiglio Nazionale delle Ricerche, Firenze, Italy; \\ e-mail: maselli@ibimet.cnr.it \\ fgeoLAB, Dipartimento di Scienze e Tecnologie Ambientali Forestali, University of \\ Firenze, Italy \\ §LAMMA, Regione Toscana, Firenze, Italy \\ -Dipartimento di Scienze dell'Ambiente Forestale e delle sue Risorse, University of \\ Tuscia, Italy \\ **Dipartimento di Scienze e Tecnologie per l'Ambiente e il Territorio, University of \\ Molise, Italy
}

(Received 22 July 2003; in final form 7 March 2005)

\begin{abstract}
Routinely, applications of nonparametric estimation methods to satellite data for assisting the creation of forest inventories in Northern European countries are stimulating interest in the possible extension of these methods to more complex Mediterranean areas. This is the subject of the current work, which presents an experiment based on the integration of remotely sensed images and sample field measurements aimed at producing forest attribute maps in central Italy. Testing was carried out in an area where 370 geocoded field plots, sampled on a singlestage cluster design, were collected to characterize wood and non-wood forest attributes. These ground data served to apply various k-Nearest Neighbour (k$\mathrm{NN}$ ) estimation procedures to multitemporal Landsat $7 \mathrm{ETM}+$ images in order to map major forest attributes (basal area and simulated leaf area index, LAI). More specifically, the investigation focused on evaluating the effects of using satellite images from different periods of the growing season and spectral metrics of increasing complexity. The results achieved by the examined methods are finally discussed in order to provide guidelines for possible operational utilization.
\end{abstract}

\section{Introduction}

Monitoring programmes are recognized as a major source of information to support the modelling and management of forest resources. In particular, forest attribute maps (e.g. species composition, vertical structure, biomass, stand height, number of trees) are important data sources for many modelling tasks such as: carbon sink evaluation, animal habitat suitability assessment and landscape biodiversity estimation (Waring and Running 1998). Traditional sample-based inventories are able to provide a proper statistical assessment of forest attributes, but, given the usual sampling intensities, they are expensive and time consuming (Corona et al. 2002). Satellite remote sensing techniques are therefore seen as a valuable source of information for mapping forest attributes (tree species composition, stand biomass, stand density, etc.) which are linked to relevant 